\title{
Recent Trends and Characteristics Associated with Influenza Vaccination Disparities among Texas Children
}

\author{
Lloyd B. Potter, $\mathrm{PhD}^{\mathrm{a}}$, Corey S. Sparks, $\mathrm{PhD}^{\text {a b }}$, Bradley Pollock, $\mathrm{PhD}^{\mathrm{c}}$,
}

Affiliations: 'Department of Demography, the University of Texas at San Antonio; ${ }^{b}$ Department of Epidemiology and Biostatistics, the University of Texas Health Science Center at San Antonio; and ${ }^{\mathrm{C} D e p a r t m e n t ~ o f ~ P u b l i c ~ H e a l t h ~ S c i e n c e s, ~ U n i v e r s i t y ~ o f ~}$ California at Davis

Corresponding Author. Lloyd Potter, University of Texas at San Antonio, Department of Demography, 501 West Cesar E. Chavez Blvd., San Antonio, Texas, 78282, (210) 4586530, Lloyd.Potter@UTSA.edu

Short title Texas Childhood Influenza Vaccination Disparities

Financial disclosure: The authors have no financial relationships relevant to this article to disclose.

Funding source: Initial phases of the research presented in this paper were supported by the San Antonio Life Sciences Institute.

Conflict of Interest Statement: The authors have no conflicts of interest relevant to this article to disclose.

What is known on this subject: Influenza vaccination for young children is recommended. However there are differentials in vaccination coverage by race and ethnicity. Little is known about the factors associated with these differentials or with changes in vaccination coverage.

What this study adds: Texas' population is large and growing quickly. This growth is being driven by increases in the Hispanic population. Focusing on race/ethnic specific trends of influenza vaccination among children permits identification of maternal and program participation characteristics associated with influenza vaccination. 


\section{Contributors' Statement:}

Lloyd Potter conceptualized the initial study, made substantial contributions to writing the manuscript, reviewed and revised the manuscript, and approved the final manuscript as submitted.

Corey Sparks carried out the analyses, made substantial contributions to writing the manuscript, reviewed and revised the manuscript, and approved the final manuscript as submitted.

Bradley Pollock contributed to the design of the analysis of the study, reviewed the statistical methodology applied, interpretation of results, reviewed the manuscript, and approved the final manuscript as submitted. 


\begin{abstract}
Background: Adequate immunization of children protects them common infections and may serve as an indicator of access to health care. Racial and ethnic differentials in immunization of children may suggest differentials in access to health care. This research describes racial and ethnic differences in childhood influenza immunization coverage and identifies social and economic characteristics associated with these immunization differentials in Texas.

Methods: Using data from the National Immunization Survey racial and ethnic differences in seasonal influenza immunization among children is examined as related to social and economic characteristics of children in Texas over the period of 2004 to 2013.

Results: Findings suggest the presence of expected differences in childhood seasonal influenza immunization for Hispanic and non-Hispanic black children compared to nonHispanic white children. Education and marital status of the mother are predictors of influenza immunization as is participation in WIC.

Conclusions: Implications of findings suggest the need for qualitative research to better understand barriers to immunization that differentially affect minority children in Texas. Addressing racial and ethnic immunization differentials among children may potentially result in reductions in other racial and ethnic health disparities as they age.
\end{abstract}




\section{Introduction}

Immunizations have been a keystone of pediatric preventive care for years, especially for infectious disease prevention in infants and young children. Adequate immunization of young children according to the required recommendations has been identified to help protect the children from many common infections. In recent years the U.S. has sustained high and relatively stable levels of childhood immunization in its quest to achieved one of the overarching goals of the Healthy People 2020 to increase immunization coverage among all children ${ }^{1}$. As immunization begins at such young ages and because it relies on access to health care, differentials in immunization coverage by race and ethnicity may be an indicator of other health related differentials as children age.

Recent work has detailed the differences in overall vaccination coverage of US children $^{2,3}$. Other studies ${ }^{4}$ show high rates of immunization coverage in US children, when using the 4:3:1:3:3:1 vaccine series. Even though there are generally high levels of total coverage, Zhao and colleagues found significant disparities by race/ethnicity and poverty level $\stackrel{2}{2}$. Hispanic children have been shown to have lower vaccination rates than non-Hispanic white children ${ }^{5}$. Despite our knowledge of these disparities in the 4:3:1:3:3:1 vaccination series, less work has been devoted to understanding disparities in seasonal influenza vaccination children ${ }^{6,7}$. Estimates of influenza vaccination among young children (6-23 months) a decade ago indicated the presence of racial and ethnic and socioeconomic disparities in coverage ${ }^{8}$. More recent estimates suggest that influenza vaccination rates among children between the ages of 6 months and 17 years have been increasing and that racial and ethnic differences are minimal ${ }^{2}$. Bhat and colleagues $\frac{10}{2}$ document that influenza death rates are highest among children under age 1, and they 
highlight the importance of influenza vaccinations in young children. In addition the CDC recommends children as young as six months be vaccinated against seasonal influenza.

Between 2010 and 2014, Texas had the second fastest population growth rate and the highest total numerical gain ${ }^{11}$. In addition, Texas has the second largest Hispanic

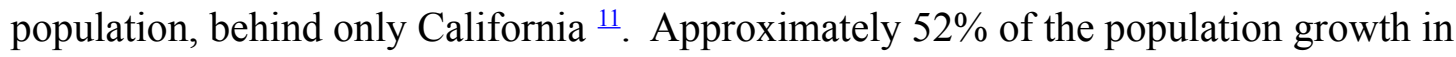
Texas between 2010 and 2014 can be attributed to growth of the Hispanic population and among persons aged 19 years and less, almost $77 \%$ of the growth is the result of population increases among Hispanics ${ }^{11}$. Texas ranks twelfth in the nation in terms of the

percent of children living in poverty ${ }^{12}$. Both of these factors put children at risk of lower vaccination coverage for the 4:3:1:3:3:1 vaccination series ${ }^{3,13}$.

Much of the rapid population growth is being driven by natural increase (birthsdeaths) in Texas, and many of these net births are in Hispanic families. This combined with the lack of published work detailing disparities within the State in seasonal influenza vaccination coverage provides is the motivation for this study. Thus we examine the disparities in Texas in seasonal influenza vaccination coverage among younger children of various racial and ethnic and income groups between 2004 and 2013.

\section{Methods}

\section{Data}

We use data from the public use data files of the National Immunization Survey (NIS) from 2004 to 2013 for this study. This NIS is conducted annually by the Centers for Disease Control and Prevention to estimate vaccination coverage rates among children 
between the ages of 19 and 35 months. The survey relies on a random digit dialing telephone survey to screen households with children, and a follow-up mail survey of the child's immunization provider. Further details of the NIS survey protocol and sampling methods can be found elsewhere ${ }^{14}$.

The outcome variable we examine is whether the respondent's child had received a seasonal influenza in during the year when the survey was conducted. Children who were not age-eligible for the vaccination were eliminated from the analytical dataset. Our focus is upon understanding patterns of disparities in Texas; therefore only children residing in Texas at the time of the survey were included in the analysis.

We consider three race/ethnic groups and two income groups. The race/ethnic categories are measured in the NIS by a respondent self-report. These groups are NonHispanic White (NHW), Non-Hispanic Black (NHB) and Hispanic. The "Other" racial group has too few cases for meaningful analysis and is not included in this analysis. To construct income groups, the NIS poverty status variable is used and dichotomized into those below poverty and those above poverty. Other maternal and household characteristics considered include maternal education, maternal age and family WIC participation. Maternal education is operationalized as two dummy variables indicating whether the child's mother had less than or greater than a High School education, with a High School education being the reference group. Maternal age is likewise operationalized as two dummy variables indicating if a child's mother was less than 19 years old, 20 to 29 years old or older than 30 (the reference group). Finally, previous research on vaccination in low income children has pointed to the use of the Supplemental Nutrition Program for Women, Infants and Children (WIC) as one 
mechanism to increase vaccination coverage ${ }^{15,16}$. This is included in the analysis using WIC usage in the past year as a predictor.

\section{Data analysis}

To examine vaccination disparities by race/ethnicity and poverty status, two analytical approaches are used. First, a stratified rate comparison between racial/ethnic and income groups in each of the years in the study period is conducted. This analysis is designed to highlight the population trends in influenza vaccination across the study period. To do this, yearly vaccination rates for each race/ethnicity and poverty status group are calculated using the survey library ${ }^{17}$ in $\mathrm{R}^{\frac{18}{}}$. This is used to estimate the proportions of each ethnic and income group that had a seasonal influenza vaccination, taking into account the stratified nature of the NIS sampling design, and appropriate population sampling weights. Once these yearly rates are calculated, temporal trend analysis using a robust linear regression model is used to visualize the observed trend in these state-level rates across the time period.

The second analytical method is a survey design corrected logistic regression model. This model is constructed to test for the effects of race/ethnicity, net of maternal characteristics and poverty status. The model is specified in three stages, the first considering only the effects of race/ethnicity on the log-odds of having been vaccinated, the second model adds the effects of maternal characteristics to the model, and the third specification adds the effects of household socioeconomic conditions to the model. Again, the survey library in R is used to estimate all model coefficients once survey design effects and sampling weights have been included. 


\section{Results}

Table 1 provides the seasonal influenza rates for each racial/ethnic and socioeconomic group. The rates for some groups were not able to be estimated due to lack of cases in the data. On average, the influenza vaccination rates in Texas have increased over the study period. Figures 1 and 2 demonstrate the temporal trend analysis of the rates from Table 1.

$<$ Table 1 about here $>$

Figure 1a illustrates the gap between NHW and NHB children, not in poverty, in immunization coverage over the period. There is a significant gap in coverage rates between NHW and NHB children, and this gap persists over most the period, but declines toward the end. When NHB children in poverty are compared to NHW children living in poverty, Figure 1b, we see that at the beginning of the period NHB children have higher rates of immunization than NHW. However by the end of the period, the rates of immunization of these children have converged and NHW rates appear to be improving more rapidly than NHB.

$<$ Figure 1a-1d about here $>$

Figure 1c illustrates the comparison between NHW and Hispanic children both living above and below (1d) the poverty line. Figure 1c shows again, that both groups living above the poverty line have increasing vaccination coverage over the period, but there is a difference between the NHW and Hispanic children immunization for most of the period favoring NHW children. Figure 1d illustrates no significant differences between 
NHW children below poverty compared to Hispanic children living below poverty. Both groups appear to have improving vaccination coverage over the period.

Table 2 presents the results of the individual-level analysis of influenza vaccination. Three models are presented: a base model with only time and race/ethnicity, a second model which adds the effects of mother's characteristics, and a final full model which controls for poverty level of the household and WIC usage. Likelihood ratio tests are shown to examine model improvement across the three models. Similar to the results from the aggregate, data, there is an average increase in the odds of a child having received the influenza vaccine over time (16\% higher odds in each year after 2004), and significant disparities between Hispanic and NHB children, when compared to whites. Hispanic children had 53\% (95\% CI 62\%-42\%) lower odds of receiving the vaccine, and NHB children had 59\% (95\% CI 69\%-41\%) lower odds than NHW children. When mother's characteristics are controlled, the disparities still persist, but diminish with Hispanic children having 37\% lower (95\% CI 50\%-20\%) and NHB children having $43 \%$ lower (95\% CI 59\%-21\%) odds than NHW children of having received the vaccine. The largest impact of the maternal control variables is mother's education, where children with a mother with more than a high school education had a 65\% higher (95\% CI 25\%$110 \%$ ) odds of receiving the vaccine compared to a child whose mother only had a high school education. The final model shows that after controlling for household poverty status and WIC usage, the disparities between the race/ethnic groups become insignificant, despite the effect of WIC reducing the odds of being vaccinated. This relationship is problematic, however, since WIC is a means-based program, and there is a high correlation between using WIC and being below the poverty line. To tease this 
collinearity apart and to examine how WIC affects the odds of immunization in the racial/ethnic minority groups, two subsequent models are fit, one considering just the impacts of poverty and WIC separately, and the other considering the interaction between WIC use and race/ethnicity.

$<$ Table 2 about here $>$

When each term is considered separately, as seen in Table 3, it is evident that the two predictors have different impacts on the racial/ethnic disparities. The poverty variable has the expected impact of lowering the odds of vaccination when taken by itself, and the disparities between NHW and Hispanics and NHB are maintained but reduced. When WIC is considered by itself, the disparities between both Hispanics and NHW become insignificant, although the effect of WIC itself is still to lower the odds of immunization. The interaction term between WIC and race/ethnicity shows that the when Hispanic and NHB children participate in WIC, the odds of receiving seasonal influenza vaccine are increased (while only marginally for Hispanic children) relative to NHW children, and for NHB children the effect is particularly significant.

$<$ Table 3 about here $>$

\section{Discussion}

Results from other authors have described how vaccination coverage in the US has increased over the last decade ${ }^{2,3}$, and how for some groups, disparities in general vaccination rates have been maintained, while others have decreased. Little work has 
been done describing how these disparities function in terms of seasonal influenza vaccination in children, although some work has described these effects ${ }^{6,9,15,19,20}$ and some. Still, these results are generally presented for the entirety of the US, and little has been done focusing on regional or state-specific analyses, despite the fact that local and state policies toward vaccination are often key determinants of their use.

Using data from the National Immunization Survey we examined the temporal trend in seasonal influenza vaccination disparities among children between the ages 19 and 35 months who are in the main race/ethnic groups in Texas. Results point to how NHB and Hispanic children continue to have lower influenza vaccination rates than their NHW peers, and that children in these racial/ethnic groups who live below poverty face additional disadvantages in being vaccinated for influenza when compared to NHW children not living in poverty. The explanation of the mechanism by which these disparities are maintained in Texas, as in many areas of the country, is likely multifaceted. As described in the national setting ${ }^{4}$, accessibility to adequate healthcare for poor populations is often an issue that is difficult to overcome, likewise discrimination based on race/ethnicity ${ }^{21}$ or language ${ }^{22,23}$ may also play a role in many circumstances ${ }^{24}$. Conceptual models of health disparities, highlight that factors influencing such disparities are often multi-level in nature. The effects of being a minority, or living below the poverty are only compounded by the physical surrounding and residential location ${ }^{25,26}$. Without data that would allow for these issues to be operationalized, we will lack clarity about the primary mechanisms for development and maintenance of such disparities.

Our finding that participating in certain programs, notably WIC, may suggest one mechanism by which minority racial/ethnic groups can overcome the disparity in 
immunization coverage $\mathrm{e}^{15,16}$. This effect is particularly significant in Texas, when compared to the rest of the US (results not shown, but available from the authors), where the effects of WIC participation significantly reduces the odds of vaccination, the same interactive effects are not found, and the disparities between NHW, Hispanics and NHB are still observed. Thus access to and involvement in some programs appears to improve the probability that younger children will become vaccinated for influenza. One study found that vaccination programs in schools resulted in higher rates of vaccination for children and suggests that institutional based vaccination efforts may be more effective than those that rely on primary health care settings $\stackrel{27}{ }$.

A better understanding of the racial and ethnic disparities in childhood seasonal influenza vaccination may be needed to develop efforts to increase coverage among minority children in Texas. Conducting qualitative research among parents may provide insight regarding the racial and ethnic differences we see in vaccination coverage. Knowing more about the barriers that parents experience when it comes to vaccination of their children that differ by race and ethnicity may lead to the development and implementation of efforts to reduce vaccination disparities. Additionally, such knowledge may also lead to better insight into strategies to reduce other race and ethnic health disparities.

\section{References}

1. U.S. Department of Health and Human Services. Healthy People 2020. http://www.healthypeople.gov/. Published 2015. 
2. Zhao Z, Luman ET. Progress Toward Eliminating Disparities in Vaccination Coverage Among US Children, 2000-2008. American Journal of Preventive Medicine. 2010;38(2):127-137.

3. Zhao Z, Smith PJ, Luman ET. Trends in early childhood vaccination coverage: Progress towards US Healthy People 2010 goals. Vaccine. 2009;27(36):50085012.

4. $\quad$ Chu SY, Barker LE, Smith PJ. Racial/ethnic disparities in preschool immunizations: United States. 1996-2001. American Journal of Public Health. 2004;94(6):973-977.

5. Darling NJ, Barker LE, Shefer AM, Chu SY. Immunization coverage among Hispanic ancestry, 2003 National Immunization Survey. American Journal of Preventive Medicine. 2005;29(5):421-427.

6. Schneider EC, Cleary PD, Zaslavsky AM, Epstein AM. Racial disparity in influenza vaccination - Does managed care narrow the gap between African Americans and whites? Jama-Journal of the American Medical Association. 2001;286(12):1455-1460.

7. Verani JR, Irigoyen M, Chen S, Chimkin F. Influenza vaccine coverage and missed opportunities among inner-city children aged 6 to 23 months: 2000-2005. Pediatrics. 2007;119(3):E580-E586.

8. Santibanez TA, Santoli JM, Bridges CB, Euler GL. Influenza vaccination coverage of children aged 6 to 23 months: The 2002-2003 and 2003-2004 influenza seasons. Pediatrics. 2006;118(3):1167-1175.

9. Santibanez TA, Peng-Jun LU, O'Halloran A, Meghani A, Singleton JA, Grabowsky M. Trends in Childhood Influenza Vaccination Coverage-U.S., 2004-2012. Public Health Reports. 2014;129(5):417-427.

10. Bhat N, Wright JG, Broder KR, Murray EL, Greenberg ME, Glover MJ, et al. Influenza-associated deaths among children in the United States, 2003-2004. New England Journal of Medicine. 2005;353(24):2559-2567.

11. U.S. Census Bureau. Annual Estimates of the Resident Population by Sex, Race, and Hispanic Origin: April 1, 2010 to July 1, 20142015.

12. U.S. Census Bureau.. 2013 1-Year American Community Survey.

13. Fiscella K. Commentary - Anatomy of racial disparity in influenza vaccination. Health Services Research. 2005;40(2):539-549.

14. NORC. A User's Guide for the 2009 Public-Use Data File. Washington, D.C.: National Center for Health Statistics; 2010.

15. Shefer A, Smith PJ. Improving the immunization and health status of children in the Women, Infants, and Children (WIC) Program. $J$ Health Care Poor Underserved. 2004;15(1):127-140.

16. Bardenheier BH, Yusuf HR, Rosenthal J, Santoli JM, Shefer AM, Rickert DL, et al. Factors associated with underimmunization at 3 months of age in four medically underserved areas. Public Health Rep. 2004;119(5):479-485.

17. Lumley T. Analysis of complex survey samples. Journal of Statistical Software. 2004;9(1):1-19.

18. R Development Core Team. R: A language and environment for statistical computing. Vienna, Austria: R Foundation for Statistical Computing; 2011. 
19. Winston CA, Wortley PM, Lees KA. Factors associated with vaccination of medicare beneficiaries in five U.S. communities: Results from the racial and ethnic adult disparities in immunization initiative survey, 2003. J Am Geriatr Soc. 2006;54(2):303-310.

20. Link MW, Ahluwalia IB, Euler GL, Bridges CB, Chu SY, Wortley PM. Racial and ethnic disparities in influenza vaccination coverage among adults during the 2004-2005 season. Am J Epidemiol. 2006;163(6):571-578.

21. May T, Silverman RD. Free-riding, fairness and the rights of minority groups in exemption from mandatory childhood vaccination. Hum Vaccin. 2005;1(1):12-15.

22. Logan JL. Disparities in influenza immunization among US adults. J Natl Med Assoc. 2009;101(2):161-166.

23. Flores G, Vega LR. Barriers to health care access for Latino children: a review. Fam Med. 1998;30(3):196-205.

24. Herrera GA, Zhao Z, Klevens RM. Variation in vaccination coverage among children of Hispanic ancestry. American Journal of Preventive Medicine. 2001;20(4):69-74.

25. Aday LA. Establishment of a conceptual base for health services research. $J$ Health Serv Res Policy. 2001;6(3):183-185.

26. Aday LA. Health status of vulnerable populations. Annu Rev Public Health. 1994;15:487-509.

27. Kansagra SM, Papadouka V, Geevarughese A, Hansen MA, Konty KJ, Zucker JR. Reaching Children Never Previously Vaccinated for Influenza Through a SchoolLocated Vaccination Program. American Journal of Public Health. 2014;104(1):e45-e49. 